\title{
Wavelet Transforms for Compound Nerve Action Potential Analysis
}

\author{
A. G. Ramakrishnan and R. V. S. Sastry \\ Biomedical Engineering Laboratory, Department of Electrical Engineering, Indian Institute of Science, Bangalore -- 560012
}

ABSTRACT

An attempt has been made to characterize Compound Nerve Action Potentials from normals and Hanseniasis patients using wavelet transforms. The work reported here involves classification of peripheral potentials recorded at the elbow of normals and Hanseniasis patients following electrical stimulation of the median nerves. Employing the system developed by the first author, nerve conduction data has been obtained from 8 normals and 8 Hanseniasis patients. The neuro-physiological data so collected was preprocessed to normalize the energy of the various responses and was analyzed using the Discrete Wavelet Transform. A new parameter, defined in the transform domain and termed as Intra-scale Inter-epoch Energy Ratio, could clearly demarcate the normals and the Hanseniasis patients at a particular scale.

Keywords : Compound nerve action potential, wavelet transform, Hanseniasis, Intra-scale Inter-epoch Energy Ratio, scalogram.

\section{INTRODUCTION}

Hansens's disease is primarily a neuropathy. Studies on nerve conduction velocities (NCV) have been reported in the affected nerves of patients. While a general reduction in NCV has been observed, there is an overlap between the ranges of NCVs for normal nerves and those affected by Hanseniasis, and thus NCVs by themselves cannot discriminate between normal and Hanseniasis nerves. It is observed that the responses are generally smooth in the case of normals, whereas for patients, a number of tiny peaks are observed after the dominant response [see Fig. la]. The average Fourier spectra of the patient responses showed distinct peaks at specific frequencies not present in the average Fourier spectra of the normal responses [1]. However, these peaks could not always be isolated in the case of individual patient spectra and thus, classification could not be achieved based on these spectra. Hence, it is thought that a method of analysis that looks at the time distribution of energies at different frequency bands could possibly distinguish between normal and abnormal waveforms. Among the Time-Frequency Representations (TFR) which could be used for this purpose, the wavelet transform introduced by Grossman and Morlet [2] is seen to be the most appropriate. The Discrete Wavelet Transform (DWT) has also been successfully used in many other biomedical applications such as ECG signal compression [3], late potential detection [4] and evoked potential analysis [5].

Proceedings RC IEEE-EMBS \& 14th BMESI - 1995

2.70

\section{METHOD}

Data acquisition employed a custom built evoked potential system [6]. Median nerves of 8 normal subjects and 8 patients were electrically stimulated at the wrist to evoke a minimal thumb twitch. Responses were recorded from the elbow at a location medial to the brachial artery. Each recorded waveform was an average of 16 responses of $20 \mathrm{~ms}$ duration digitized at a rate of $16.67 \mathrm{kHz}$. For further processing, a window of length 256 samples was selected from each response and. scaled to normalize its energy.

The goal of the wavelet transform is to decompose any arbitrary signal $f(x)$ into a summation of wavelets at different scales and shifts as given below.

$$
f(x)=a_{0}+\sum_{j} \sum_{k} a_{2^{j}+k} W\left(2^{j} x-k\right) ; 0 \leq x<1
$$

The integer $j$ denotes different levels of wavelets, starting with $j=0$; integer $\mathrm{k}$ covers the number of wavelets in each level, namely from $k=0$ to $2^{\prime}-1$. The DWT is an algorithm for computinga,,$_{-k}$, when $f(x)$ is sampled at equally spaced intervals over $0 \leq x<1$. The DWT algorithm was discovered by Mallat [7] and is therefore also known as Mallat's Pyramid algorithm. As the number of the wavelet coefficients increases, a wavelet becomes smoother and resembles a smoothly windowed harmonic function. Daubechies wavelet of 8 taps [8] was found to be suitable for our analysis. The scalogram or time-frequency energy distribution of $f(x)$ at various levels is obtained by squaring eq. (1) and is given by

$$
\int_{0}^{1} f^{2}(x) d x=a_{0}^{2}+\sum_{j} \sum_{k} a_{2^{j}+k}^{2}\left(\frac{1}{2^{j}}\right)
$$

When comparing energies across various levels/scales, it is important to take care of the scaling factor $\left(1 / 2^{j}\right)$, which is dependent on the wavelet level $j$. However, the current study examined the distribution of signal energy across time epochs within each scale. For this, the signal at each scale was divided into two equal epochs and the ratio of the energy in the first half of the signal to that in the second half was obtained for each scale. This ratio, designated as Intra-scale Inter-Epoch Energy Ratio at level $j\left(\right.$ IIER $\left._{j}\right)$ is defined as,

$$
I I E R_{j}=\sum_{k=0}^{2^{j-1}-1} a_{2^{j}+k}^{2} / \sum_{k=2^{j-1}}^{2^{j}-1} a_{2^{j}+k}^{2}
$$




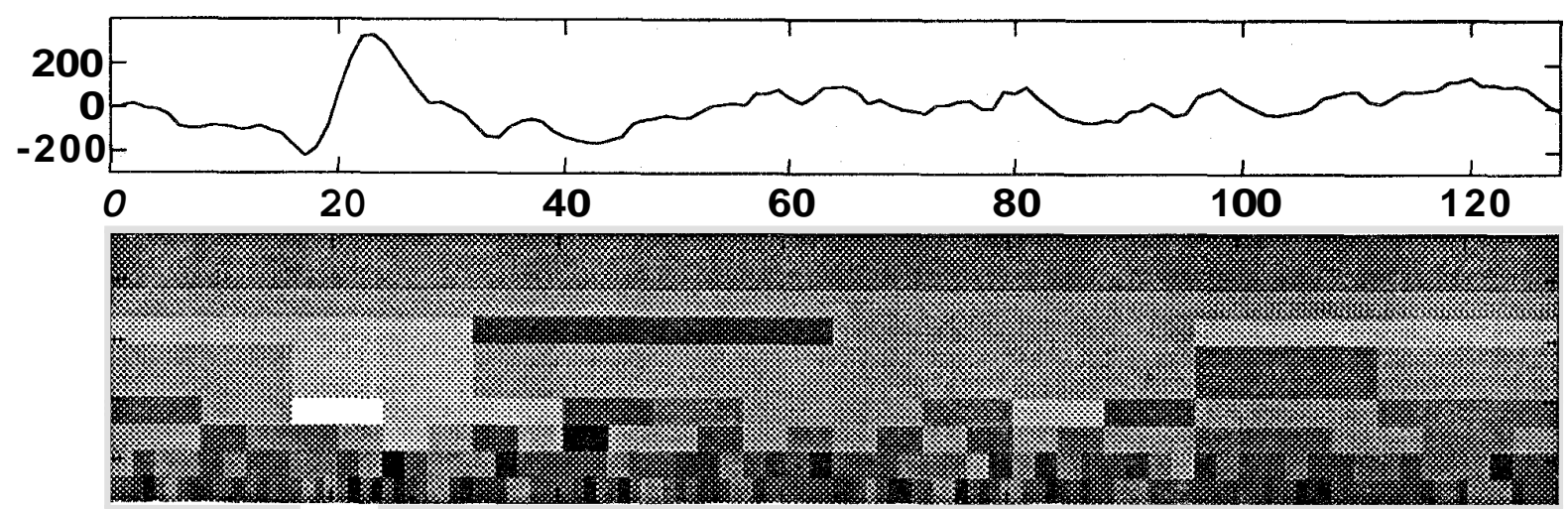

Fig. 1. A Compound Nerve Action Potential from a patient and its scalogram

\section{RESULTSAND DISCUSSION}

Figs. $1 \mathrm{a}$ and $1 \mathrm{~b}$ show, respectively, a sample waveform from a patient and its scalogram represented as a gray scale image with eight levels of quantization. The dominant peak in the waveform gives rise to the bright segment seen in the initial portion of scale 5 .

Figs. $2 \mathrm{a}$ and $2 \mathrm{~b}$ show the values of the IIER at scales 5 and 3 respectively for all the normals and patients. As there was a wide difference between the ranges of values of IIER for normals and patients at scale 5 , the logarithm of this ratio has been plotted. It can be clearly seen that the ratio is much higher for normals than for patients at this scale. On the other hand, there is a complete overlap and consequently, absence of any observable pattern between the values for the two groups at scale 3 . These scales correspond roughly to frequency bands of $520-1040 \mathrm{~Hz}$ (scale 5) and 130 to $260 \mathrm{~Hz}$ (scale 3). Thus the value of IIER s $_{5}$ could be used to distinguish a normal nerve from a pathological nerve affected by Hanseniasis.


Fig. 2. $\log _{10}$ (IIER) of subjects (a) at scale 5 and (b) at scale 3.

Proceedings RC IEEE-EMBS \& 14th BMESI - 1995

\section{CONCLUSION}

The application of DWT has facilitated the extraction of the relevant information from the data. It is clear that IIIRR at different scales has the potential to distinguish between normal and abnormal responses in Hanseniasis. Spectral estimation based on techniques such as DCT or DFT do not give information on the time intervals where energy at a particular frequency band is concentrated and thus cannot achieve classification of above data. Further studies on more data can reveal the utility of the above technique in serial evaluation of affected nerves for prognosis and for testing the effectivenessof treatment.

\section{REFERENCES}

1. Srinivasan T M and Ramakrishnan A G (1992) "Central Conduction in Leprosy - a Multidomain Study," Proceedings of the 14th Annual International Conference of the IEEE EMBS, Paris, France, 14,2491-2492.

2. Grossman A and Morlet J (1984) "Decomposition of Harr functions into square integrable wavelets of constant shape," SLAM J. Math., 15, 723-736.

3. Sastry R V S and Rajgopal K (1995) "ECG Signal Compression using Wavelets," accepted for the Regional Conference of IEEE EMBS, New Delhi, Feb. 15-18.

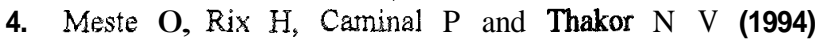
"Ventricular Late Potentials Characterization in Time Frequency Domain by Means of a Wavelet Transform," IEEE Trans. BME, 11,1085-1093.

5. Sita G, Ramakrishnan A G and Sastry R V S (1995) "Time Varying Filter for Estimation of Evoked Potentials," accepted for the Regional Conference of IEEE EMBS, New Delhi, Feb.

6. Ramakrishnan A G (1989) Evoked Potential Monitoring and Analysis in Health and Disease, I. I. T., Madras.

7. Mallat S (1989) “A Theory for Multiresolution Signal Decomposition : The Wavelet Representation," IEEE Trans. PAMI, 11, 674-693.

8. Daubechies I (1988) "Orthonormal bases of compactly supported wavelets,” Comm.Pure Appl. Math., 41,909-996. 